\title{
Secernin 1 promotes cell invasion and migration by activating the TGF- $\beta /$ Smad3 pathway in oral squamous cell carcinomas
}

\section{Li Xiao}

North Sichuan Medical University

\section{Shuqiong Wen}

Sun Yat-Sen University

Qianyu Zhang

North Sichuan Medical University

Yanshuang Peng

North Sichuan Medical University

Kaiyue Zheng

North Sichuan Medical University

Lianjie Yang

North Sichuan Medical University

Zhi Wang

Sun Yat-Sen University

Ying Liu ( $\nabla$ liuying08_nsmz@163.com )

North Sichuan Medical University https://orcid.org/0000-0003-0917-9716

\section{Research article}

Keywords: SCRN1, OSCC, invasion, metastasis, TGF- $\beta$ /Smad3 pathway

Posted Date: January 27th, 2020

DOI: https://doi.org/10.21203/rs.2.21920/v1

License: (c) (1) This work is licensed under a Creative Commons Attribution 4.0 International License. Read Full License 


\section{Abstract}

\section{Background}

Secernin-1 (SCRN1) is a regulator of exocytosis in mast cells. Recently, SCRN1 has been reported to be correlated with the prognosis of colorectal cancer and gastric cancer but its functional effects on oral squamous cell carcinoma (OSCC) remains unclear. Our aim was to explore the expression pattern and the migration and invasion effects of the newly identified SCRN1 in OSCC.

\section{Methods}

Western blotting (WB) was used to measure SCRN1 expression in human OSCC tissue samples and OSCC cell lines. Then, the effects of SCRN1 on OSCC cell proliferation, invasion and metastasis were analyzed by cell counting kit-8 (CCK-8) and transwell assays. The secretion of matrix metalloproteinase (MMP)-2 and MMP-9 in SCRN1 knockdown OSCC cells and vector cells was investigated by enzymelinked immunosorbent assay (ELISA). The expression levels of TGF- $\beta$, Smad3 and phosphorylated Smad3 (p-Smad3) in SCRN1 knockdown OSCC cells and vector cells were measured by WB.

Results

The expression of SCRN1 was significantly elevated in the OSCC tissues and cell lines. Furthermore, SCRN1 knockdown attenuated the proliferation, migration and invasion of SCC15 and HSC3 cells. SCRN1 knockdown reduced the secretion of MMP-9 from HSC3 and SCC15 cells, but the secretion of MMP-2 did not obviously change. Additionally, SCRN1 knockdown reduced the expression of TGF- $\beta$ and p-Smad3 in HSC3 and SCC15 cells.

\section{Conclusions}

Our study demonstrated that SCRN1 is upregulated in OSCC. Further studies demonstrated that SCRN1 promotes proliferation, invasion and metastasis of OSCC cells via TGF- $\beta /$ Smad3 signaling.

\section{Introduction}

Oral squamous cell carcinoma (OSCC) accounts for approximately $90 \%$ of all malignancies in the oral cavity, and it is the sixth most common cancer worldwide with over 200,000 newly diagnosed patients each year[1, 2]. Management of OSCC remains surgical, with adjuvant radiotherapy or chemoradiation reserved for advanced stage tumors[3]. The 5-year survival rate for OSCC in most countries is approximately $50 \%$ [4]. Approximately $30-50 \%$ of OSCC patients have metastatic lymph nodes during clinical examination, which are associated with poor outcome[5]. Due to its higher lymph node metastasis rate, low cure rate and high mortality, OSCC represents a global public health problem with a great individual and socioeconomic burden. However, the underlying mechanism of the pathogenesis and metastasis of OSCC is unclear. 
Secernin-1 (SCRN1) is a newly identified cytoplasmic protein, which was originally shown to regulate the exocytosis of mast cells[6]. The expression of SCRN1 in gastric cancer tissues is upregulated according to CDNA microarray expression profiles, and SCRN1 is a new immunotherapeutic target[7]. A previous study has suggested that upregulated SCRN1 expression is linked to poor prognosis and metastasis in colon cancer[8], but the opposite effect has been reported in synovial sarcoma[9]. SCRN1 serves as a positive prognostic marker for synovial sarcoma with significantly higher expression among patients who have survived without metastasis for least 5 years[10]. However, the expression pattern, functional effects and underlying molecular mechanism of SCRN1 in the progression of OSCC remain unknown.

Exocytosis is the process by which the contents of vesicles are released into the extracellular matrix via the fusion with cytoplasmic membrane. Cancer cells deliver surface proteins and release soluble factors (growth factors and cytokines) through exocytosis into the environment facilitate the invasion and growth of tumors[11]. Increasing evidence demonstrates that exocytosis is associated with tumor growth, migration, and metastasis[12-14]. It has been reported that SCRN1 can accelerate the invasion and metastasis of colon cancer by increasing MMP-2/MMP-9 secretion[15]. The transforming growth factor (TGF)- $\beta /$ Smad3 signaling pathway plays an important role in the invasion and migration of various malignancies[16-19]. Moreover, TGF- $\beta$ induced the phosphorylation of Smad3 and regulates expression of metastasis associated genes[20]. Therefore, we hypothesized that SCRN1 promotes cell invasion and migration by activating the TGF- $\beta /$ smad3 pathway in OSCC.

\section{Materials And Methods}

\section{Patients and specimens}

This study was approved by the Ethics Committee of the North Sichuan Medical College. All specimens were collected after obtaining informed consent from patients. Primary OSCC tissue samples and matching adjacent normal mucosa specimens were collected from 15 patients undergoing surgery at the North Sichuan Medical College, China. All collected tissues were stored at $-80^{\circ} \mathrm{C}$ until use. The pathological or normal state of the samples was confirmed by histopathological and clinical examination.

\section{Cell culture and cell transfection}

Six human oral squamous cell carcinoma cell lines including HSC3, HSC6, SCC15, SCC25, UM1 and UM2, were cultured in Dulbecco's modified Eagle's medium (DMEM, HYCLONE, USA) supplemented with 10\% fetal bovine serum, $100 \mathrm{U} / \mathrm{mL}$ penicillin, and $100 \mu \mathrm{g} / \mathrm{mL}$ streptomycin, and normal oral keratinocytes (NOKs) were grown in keratinocyte growth medium. All the cells were maintained at $37^{\circ} \mathrm{C}$ in a $5 \% \mathrm{CO}_{2}$ humidified incubator.

The sh-SCRN1 plasmids and empty vector were designed and synthesized by GeneCopoeia (https://www.genecopoeia.com) and the viruses were generated using protocols from Gene-Copoeia. HSC3 and SCC 15 cells ( $2 \times 10^{5}$ /well) were seeded in 6-well plates and grown for $24 \mathrm{~h}$ until they reached 
$60-70 \%$ confluency. The cells were then transfected with diluted virus. After $12 \mathrm{~h}$, the medium was replaced with fresh complement medium and cells were harvested $72 \mathrm{~h}$ after transfection.

\section{Western blot assay}

The cells were lysed in RIPA lysis buffer (CWBIO, China) supplemented with protease inhibitors. Total proteins were fractionated using SDS-PAGE, transferred onto PVDF membranes (Millipore, USA), and examined by the corresponding primary antibodies using a chemiluminescence kit (Millipore, USA). The primary antibodies used in this study were as follows: anti-SCRN1 antibody (Abcam, ab105355, USA), anti-GAPDH antibody (Earthox, E021010, USA), anti-Smad3 antibody (HuaAn Biotechnology, ET1607, China), and anti-Phospho-Smad3 antibody (S423/S425) (HuaAn Biotechnology, ET1609, China).

\section{Quantitative real-time PCR (qPCR)}

Total RNA from cells was extracted according to the manufacturer's protocol (E.Z.N.A Total RNA Kit, Omega , USA), and total RNA was reverse transcribed to cDNA in strict accordance with the manufacturer's instructions (PrimeScript ${ }^{\mathrm{TM}}$ RT Reagent Kit, TaKaRa, Japan), which was then amplified with SYBR (Roche) by qPCR. The sequences of the primers were as follows: SCRN1 (Forward: 5'GGATGGTCTGGTGGTATTTGG-3', and Reverse: 5'-CCTTGGAACTTGGTCGATTG-3'); and GAPDH (Forward: 5'-GACTCATGACCACAGTCCATGC-3'; and reverse: 5'-AGAGGCAGGGATGATGTTCTG-3'). The relative levels of SCRN1 mRNA were normalized to GAPDH reference gene expression and calculated via the $2^{-\triangle \triangle C T}$ method.

\section{Cell counting kit-8 (CCK-8) assay}

The cells were seeded in 96-wells plates (1000 cells/well) for the cell proliferation assay (CCK-8 kit, Yeasen, China). Then $10 \mu \mathrm{l}$ of CCK-8 solution was added into each well after the cells were cultured for $24,48,72$ and $96 \mathrm{~h}$. After adding the CCK8 reagent, the cells were cultured for an additional 1-4h, and the OD values were measured at $450 \mathrm{~nm}$.

\section{Transwell migration and invasion assays}

The cells $\left(2 \times 10^{5}\right)$ were seeded into the upper chambers of a 24-well transwell plate containing serumfree medium. Medium supplemented with $10 \%$ FBS was added to the lower chambers. Before the transwell invasion assays were performed, a mixture of Matrigel (BD Biosciences, USA) and medium was spread on the upper surface of the chambers and incubated at $37^{\circ} \mathrm{C}$ overnight. The cells were then allowed to incubate for $24 \mathrm{~h}$ at $37^{\circ} \mathrm{C}$ with $5 \% \mathrm{CO}$. Cells were fixed and stained with crystal violet $(0.1 \%)$. The cells that migrated from the upper chamber to the lower chamber were counted and the average number of five random regions were considered the final result.

\section{Enzyme-Linked Immunosorbent Assay (ELISA)}


The MMP-2/MMP-9 concentrations in the supernatants were determined by ELISA using a commercially available kit (Abcam, Cambridge, UK). The assay was performed according to the manufacturer's specifications. The absorbance was read by a microplate reader at $450 \mathrm{~nm}$. The concentration of each sample was measured based on the regression equation established using serial dilutions of standards.

\section{Statistical analysis}

All the statistical analyses were performed by using GraphPad Prism 6.0 and Stata/MP 14.0. All the data are presented as the mean \pm standard deviation (SD) from three independent experiments. A two-sided $P$ $\leq 0.05$ indicated a statistical significance.

\section{Results}

\section{SCRN1 expression is significantly increased in OSCC tissues and cell lines}

SCRN1 protein expression was compared between 15 cancerous tissues and the adjacent normal mucosa. The SCRN1 expression levels in the OSCC tissues were significantly higher than those in the normal mucosa tissues. Among the 15 OSCC samples, 12 had higher SCRN1 expression compared with that in the paired normal mucosa, and the difference between the OSCC tissue and adjacent normal mucosa was statistically significant (Figure. 1a, $P<0.001$ ). Furthermore, the SCRN1 expression level was analyzed by WB analysis in the OSCC cell lines, including HSC3, HSC6, SCC15, SCC25, UM1 and UM2 (Figure. 1b). SCRN1 expression was significantly upregulated in OSCC cells compared to that in NOKs $(P<0.05)$. Taken together, these results indicated for the first time that SCRN1 is highly expressed in OSCC tissues and 6 OSCC cell lines compared to controls. Moreover, the increased expression of OSCC may be closely related to the development of OSCC.

\section{Knockdown of SCRN1 suppresses proliferation, invasion and metastasis of OSCC cell lines}

SCRN1 promotes exocytosis in cells, which regulates the secretion of several cytokines and proteins related to metastasis such as MMP-2/MMP-9, thus promoting the metastasis of tumors[15]. Therefore, we hypothesized that SCRN1 could play a role in the migration and invasion of OSCC. To investigate the role of SCRN1 in OSCC proliferation, invasion and migration, we selected two OSCC cell lines, namely HSC3 and SCC15 due to their relatively high SCRN1 expression. To establish SCRN1 stable knockdown cell lines, WB and qPCR assays were performed to identify the optimal short hairpin RNA (shRNA) from three selected putative shRNAs (shRNA-a, shRNA-b, and shRNA-c) (Figure. 2a). When HSC3 cells were transfected with shRNA-c, the majority cells died. We repeated the experiment three times and the results were remained consistent. The results indicated that shRNA-a had the highest knockdown efficiency and was therefore used to produce the knockdown cell line.

The CCK-8 proliferation assay revealed that the proliferation capacity of the OSCC cells was diminished after SCRN1 was knocked down (Figure. 2b). In the transwell migration assay, SCRN1 knockdown significantly reduced the cell migration ability in $\mathrm{HSC} 3$ cells compared to that of in the control cells 
$(P<0.05)$. Similar results were observed in the SCC15 cells, suggesting that the effect may not be cell-type specific $(P<0.01)$ (Figure. $2 \mathrm{c})$. The transwell invasion assay demonstrated that the number of invasive cells in the SCRN1 knockdown HSC3 cells was almost three times lower than that in vector cells $(p<$ 0.01 )(Figure. $2 \mathrm{~d}$ ). In the SCC15 cells, similar effects were observed $(p<0.01)$. Thus, these results showed that SCRN1 promotes cancer cell proliferation, invasion and migration in OSCC.

\section{SCRN1 enhances MMP-9 exocytosis and activates the TGF- $\beta /$ Smad3 pathway}

We further aimed to elucidate the potential molecular mechanisms by which SCRN1 contributes to the proliferation, migration and invasion of cells in OSCC. MMPs, particularly the MMP-2 and MMP-9 gelatinases, mediate degradation of the extracellular matrix, thereby facilitating the invasion and spread of OSCC cells[21]. Therefore, to further verify that SCRN1 contributes to the invasion and migration of OSCC cells, MMP-2/9 secretion was investigated in control and SCRN1-knockdown OSCC cells using ELISA. Significant inhibition of MMP-9 secretion was observed in SCRN1knockdown SCC15 and HSC3 cells compared to that in the vector cells $(p<0.01)$ (Figure. 3a), but no obvious change in the MMP-2 secretion was observed $(p>0.05)$. These results indicated that SCRN1 enhances the exocytosis of MMP9 protein but has little impact on MMP-2 exocytosis.

Since accumulating studies have confirmed that TGF- $\beta$ / Smad3 signaling exhibits extensive regulatory roles in the development and progression of multiple types of cancers, we next conducted Western blot assays to assess the effects of SCRN1 knockdown on the activity of TGF- $\beta$ / Smad3 signaling. Knockdown of SCRN1 reduced the TGF- $\beta$ and P-Smad3 levels in HSC3 cells, but it did not change the Smad3 level in HSC3 cells (Figure. 3b). Similar results were observed for the SCC15 cells (Figure. 3b). Therefore, these results indicated that the knockdown of SCRN1 suppresses the activation of the TGF$\beta /$ Smad3 pathway in OSCC cells.

\section{Discussion}

OSCC remains a major cause of morbidity and mortality in patients with head and neck cancers worldwide. Despite recent advances in treatment modalities including surgery, chemotherapy, biologic therapy, and radiotherapy, the overall survival has remained poor over the past few years[22]. Local invasion and lymph node metastasis are still important reasons for the poor prognosis of OSCC[23]. Recently, the identification of key molecular alterations in cancer has resulted in major advances in the diagnosis and targeted therapies with validated biomarkers. Therefore, the discovery of novel biomarkers for targeted therapeutics to improve the clinical survival rates of OSCC patients is urgently needed. Our study focused on the newly identified protein, SCRN1. We found that the expression of SCRN1 was highly expressed in 15 specimens of OSCC tissue and 6 OSCC cell lines, which was consistent with the expression of SCRN1 in gastric cancer and colorectal cancer[7, 8].

SCRN1 regulates mast cell exocytosis through a mechanism that has been well defined. Exocytosis is a process by which cells transport and release secretory products through the cytoplasm to the cell membrane, and this process promotes tumor growth, metastasis and invasion. Lin et al. [15] reported that 
SCRN1 expression is upregulated in colorectal cancer and that its overexpression promotes the proliferation and invasion of colorectal cancer cells. However, the function of SCRN1 in OSCC remains unknown. In this study, we performed CCK-8 and transwell assays to investigate the functions of SCRN1 in OSCC cells (HSC3/SCC15), and we found that knockdown of SCRN1 suppressed OSCC cell proliferation, migration and invasion. MMP exocytosis, especially MMP-2/9 exocytosis, is essential for tumor cell invasion and metastasis[24]. Because SCRN1 regulated exocytosis in other cell types, we speculated that SCRN1 may increase the secretion of MMP-2/9 to promote OSCC cell invasion and migration. In the present study, ELISAs demonstrated that MMP-9 secretion was suppressed in SCRN1 knockdown cells but that MMP-2 secretion was not significantly changed. These results indicated that SCRN1 promotes proliferation, migration and invasion of OSCC cells by enhancing the secretion of MMP9.

Many classical and critical signaling pathways, such as Wnt/ $\beta$-catenin, TGF- $\beta$ and PI3K/Akt/mTOR, have been involved in the regulation of tumor growth and progression[25-27]. Despite the well-known TGF- $\beta$ suppressor effect during the early stages of tumor development, there is strong evidence that the TGF- $\beta$ might later contribute to promote proliferation, invasion, angiogenesis, and oncogenesis in advanced cancer[28]. Increasing evidence has confirmed that many novel markers promote tumor progression by activating the TGF- $\beta$ pathway[29, 30]. Recently, increasing numbers of studies have reported that exocytosis-associated proteins may regulate activation of the TGF- $\beta$ pathway, thereby influencing the progression of tumors[31, 32]. However, the association between SCRN1 and TGF- $\beta / S m a d 3$ pathway remains largely unclear. To explore the potential mechanism by which SCRN1 exhibits its tumorpromotive roles in OSCC cell proliferation, invasion and metastasis, we investigated whether SCRN1 influences the TGF- $\beta$ /Smad3 pathway, which is the most common TGF pathway. The results of Western blot analysis indicated that knockdown of SCRN1 decreased p-Smad3 level, suggesting that SCRN1 may act as a tumor promoter in OSCC by modulating the TGF- $\beta /$ Smad3 pathway.

In summary, our data provided evidence, for the first time, that SCRN1 is highly expressed in OSCC tissue and that SCRN1 knockdown inhibits OSCC cell proliferation, invasion and migration. Moreover, our results showed that SCRN1 may contribute to the progression of OSCC by enhancing the secretion of MMP-9 and activating TGF- $\beta /$ Smad3 pathway. Activation of the TGF- $\beta /$ Smad 3 and MMP signaling is frequently associated with OSCC progression. In our study, both pathways were involved in SCRN1 oncogenic functions. Thus, these results suggested that SCRN1 promotes OSCC invasion and metastasis by regulating the secretion of MMP-9 through TGF- $\beta$ /Smad3 signaling, but the underlying mechanism requires further investigation. These results suggested that SCRN1 may be a target for OSCC therapy in the future.

\section{Abbreviations}

Secernin-1 SCRN1

oral squamous cell carcinoma OSCC 
Western blotting WB

cell counting kit-8 CCK-8

enzyme-linked immunosorbent assay ELISA

matrix metalloproteinase MMP

phosphorylated Smad3 p-Smad3

transforming growth factor TGF- $\beta$

normal oral keratinocytes NOKs

short hairpin RNA shRNA

\section{Declarations}

\section{Ethics approval and consent to participate}

This study was approved by the Ethics Committee of the North Sichuan Medical College.

\section{Consent for publication}

Not applicable.

\section{Availability of data and materials}

All data analyzed during this study are included in this published article.

\section{Competing interests}

The authors declare that they have no competing interests.

\section{Funding}

This work was supported by the Special Fund for Municipal and University Cooperative Scientific Research (no.: 18SXHZ0270), the Scientific Research Project of Guangdong Provincial Key Oral Medicine Laboratory (no.: KF2016120103) and the Doctor of North Sichuan Medical College Fund (no. : CBY13-QD07. The funding bodies had no role in the design of the study and collection, analysis, and interpretation of data and in writing the manuscript.

\section{Authors' contributions}

QYZ and YSP and performed the data acquisition. KYZ and LJY conducted statistical analysis. LX and SQW undertook some experiments in vitro and drafted the manuscript. $Y L$ and $Z W$ conceived the study, 
supervised the experiments, and corrected the final version of the manuscript. All authors read and approved the final manuscript.

Acknowledgements

Not applicable.

\section{References}

1. Chi AC, Day TA, Neville BW: Oral cavity and oropharyngeal squamous cell carcinoma-an update. $C A$ : a cancer journal for clinicians 2015, 65(5):401-421.

2. Jiang L, Lv L, Liu X, Jiang X, Yin Q, Hao Y, Xiao L: MiR-223 promotes oral squamous cell carcinoma proliferation and migration by regulating FBXW7. Cancer biomarkers : section A of Disease markers 2019, 24(3):325-334.

3. Huang TH, Li KY, Choi WS: Lymph node ratio as prognostic variable in oral squamous cell carcinomas: Systematic review and meta-analysis. Oral oncology 2019, 89:133-143.

4. Seki S, Fujiwara M, Matsuura M, Fujita S, Ikeda H, Asahina I, Ikeda T: Prediction of outcome of patients with oral squamous cell carcinoma using vascular invasion and the strongly positive expression of vascular endothelial growth factors. Oral oncology 2011, 47(7):588-593.

5. Subramaniam N, Balasubramanian D, Kumar N, Murthy S, Vijayan SN, Nambiar A, Vidhyadharan S, Thankappan K, lyer S: Lymph node staging systems in oral squamous cell carcinoma: A comparative analysis. Oral oncology 2019, 97:92-98.

6. Way G, Morrice N, Smythe C, O'Sullivan AJ: Purification and identification of secernin, a novel cytosolic protein that regulates exocytosis in mast cells. Molecular biology of the cell 2002, 13(9):3344-3354.

7. Suda T, Tsunoda T, Uchida N, Watanabe T, Hasegawa S, Satoh S, Ohgi S, Furukawa Y, Nakamura Y, Tahara $\mathrm{H}$ : Identification of secernin 1 as a novel immunotherapy target for gastric cancer using the expression profiles of cDNA microarray. Cancer science 2006, 97(5):411-419.

8. Miyoshi N, Ishii H, Mimori K, Sekimoto M, Doki Y, Mori M: SCRN1 is a novel marker for prognosis in colorectal cancer. Journal of surgical oncology 2010, 101(2):156-159.

9. Suehara Y, Tochigi N, Kubota D, Kikuta K, Nakayama R, Seki K, Yoshida A, Ichikawa H, Hasegawa T, Kaneko $\mathrm{K}$ et al: Secernin-1 as a novel prognostic biomarker candidate of synovial sarcoma revealed by proteomics. Journal of proteomics 2011, 74(6):829-842.

10. de Necochea-Campion R, Zuckerman LM, Mirshahidi HR, Khosrowpour S, Chen CS, Mirshahidi S: Metastatic biomarkers in synovial sarcoma. Biomarker research 2017, 5:4.

11. Hendrix A, Westbroek W, Bracke M, De Wever O: An ex(o)citing machinery for invasive tumor growth. Cancer research 2010, 70(23):9533-9537. 
12. Palmer RE, Lee SB, Wong JC, Reynolds PA, Zhang H, Truong V, Oliner JD, Gerald WL, Haber DA: Induction of BAIAP3 by the EWS-WT1 chimeric fusion implicates regulated exocytosis in tumorigenesis. Cancer cell 2002, 2(6):497-505.

13. Hendrix A, Maynard D, Pauwels P, Braems G, Denys H, Van den Broecke R, Lambert J, Van Belle S, Cocquyt V, Gespach C et al: Effect of the secretory small GTPase Rab27B on breast cancer growth, invasion, and metastasis. Journal of the National Cancer Institute 2010, 102(12):866-880.

14. Wang YS, Tzeng HT, Tsai $\mathrm{CH}$, Cheng HC, Lai WW, Liu HS, Wang YC: VAMP8, a vesicle-SNARE required for RAB37-mediated exocytosis, possesses a tumor metastasis suppressor function. Cancer letters 2018, 437:79-88.

15. Lin S, Jiang T, Yu Y, Tang H, Lu S, Peng Z, Fan J: Secernin-1 contributes to colon cancer progression through enhancing matrix metalloproteinase-2/9 exocytosis. Disease markers 2015, 2015:230703.

16. Tong $H$, Yin H, Hossain MA, Wang Y, Wu F, Dong $X$, Gao S, Zhan K, He W: Starvation-induced autophagy promotes the invasion and migration of human bladder cancer cells via TGFbeta1/Smad3-mediated epithelial-mesenchymal transition activation. Journal of cellular biochemistry 2019, 120(4):5118-5127.

17. Wang X, Lai Q, He J, Li Q, Ding J, Lan Z, Gu C, Yan Q, Fang Y, Zhao X et al: LncRNA SNHG6 promotes proliferation, invasion and migration in colorectal cancer cells by activating TGF-beta/Smad signaling pathway via targeting UPF1 and inducing EMT via regulation of ZEB1. International journal of medical sciences 2019, 16(1):51-59.

18. Jin Y, Chen W, Yang H, Yan Z, Lai Z, Feng J, Peng J, Lin J: Scutellaria barbata D. Don inhibits migration and invasion of colorectal cancer cells via suppression of PI3K/AKT and TGF-beta/Smad signaling pathways. Experimental and therapeutic medicine 2017, 14(6):5527-5534.

19. Wu TT, Lu J, Zheng PQ, Liu SL, Wu J, Sun W, Sun QM, Ma NX, Ding XL, Chen M et al: Yiqi Huayu Jiedu Decoction Inhibits the Invasion and Metastasis of Gastric Cancer Cells through TGFbeta/Smad Pathway. Evidence-based complementary and alternative medicine : eCAM 2017, 2017:1871298.

20. Massague J: How cells read TGF-beta signals. Nature reviews Molecular cell biology 2000, 1(3):169178.

21. Jacob A, Prekeris R: The regulation of MMP targeting to invadopodia during cancer metastasis. Frontiers in cell and developmental biology 2015, 3:4.

22. Gharat SA, Momin M, Bhavsar C: Oral Squamous Cell Carcinoma: Current Treatment Strategies and Nanotechnology-Based Approaches for Prevention and Therapy. Critical reviews in therapeutic drug carrier systems 2016, 33(4):363-400.

23. Safi AF, Kauke M, Grandoch A, Nickenig HJ, Zoller J, Kreppel M: The importance of lymph node ratio for patients with mandibular infiltration of oral squamous cell carcinoma. Journal of cranio-maxillofacial surgery : official publication of the European Association for Cranio-Maxillo-Facial Surgery 2018, 46(6):1007-1012. 
24. Polette M, Nawrocki-Raby B, Gilles C, Clavel C, Birembaut P: Tumour invasion and matrix metalloproteinases. Critical reviews in oncology/hematology 2004, 49(3):179-186.

25. Clevers H, Nusse R: Wnt/beta-catenin signaling and disease. Cell 2012, 149(6):1192-1205.

26. Bellomo C, Caja L, Moustakas A: Transforming growth factor beta as regulator of cancer stemness and metastasis. British journal of cancer 2016, 115(7):761-769.

27. Simpson DR, Mell LK, Cohen EE: Targeting the PI3K/AKT/mTOR pathway in squamous cell carcinoma of the head and neck. Oral oncology 2015, 51(4):291-298.

28. Seoane J, Gomis RR: TGF-beta Family Signaling in Tumor Suppression and Cancer Progression. Cold Spring Harbor perspectives in biology 2017, 9(12).

29. Syed V: TGF-beta Signaling in Cancer. Journal of cellular biochemistry 2016, 117(6):1279-1287.

30. Colak S, Ten Dijke P: Targeting TGF-beta Signaling in Cancer. Trends in cancer 2017, 3(1):56-71.

31. Qian X, Zhou X, Shentu P, Yao Y, Jiao D, Chen Q, Zhou J, Xu Y: Sec3 knockdown inhibits TGF-beta induced epithelial-mesenchymal transition through the down-regulation of Akt phosphorylation in A549 cells. Biochemical and biophysical research communications 2019.

32. Takai E, Tsukimoto M, Harada H, Sawada K, Moriyama Y, Kojima S: Autocrine regulation of TGFbeta1-induced cell migration by exocytosis of ATP and activation of P2 receptors in human lung cancer cells. Journal of cell science 2012, 125(Pt 21):5051-5060.

\section{Figures}


Supplementary figure 1
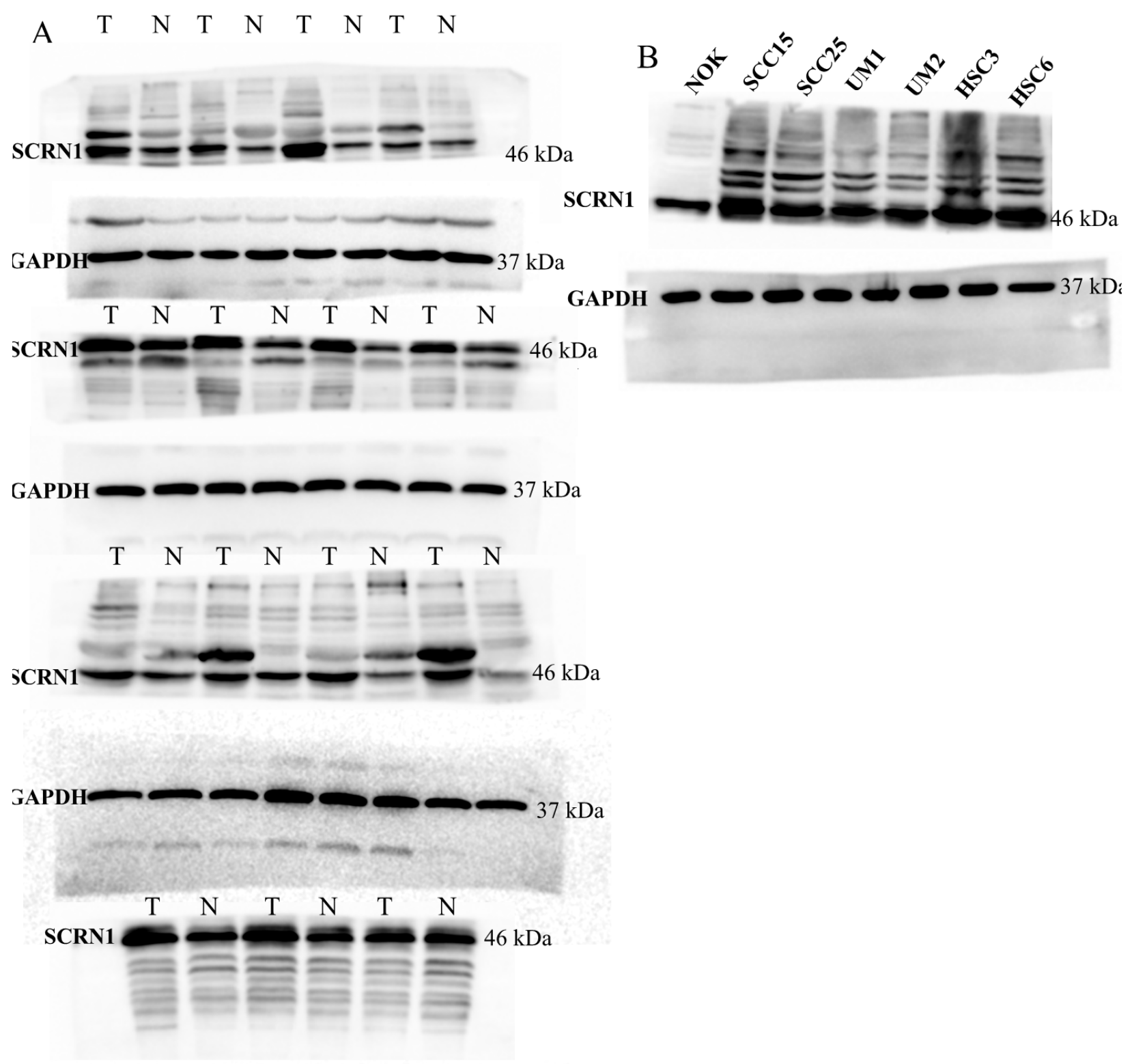

GAPDH $37 \mathrm{kDa}$

\section{Figure 1}

SCRN1 expression is significantly increased in OSCC tissues and cell lines (a) The expression levels of SCRN1 were measured in 15 paired OSCC tissues and adjacent normal mucosa by WB. Data are presented as the mean \pm SD. ${ }^{* \star *} p<0.001$. (b) The expression levels of SCRN1 were examined by WB in HSC3, HSC6, SCC15, SCC25, UM1 and UM2 cell lines. Data presented as the mean \pm SD of three independent experiments. ${ }^{*} p<0.05,{ }^{*} p<0.01$ and ${ }^{* *} p<0.001$. 
Supplementary figure 2
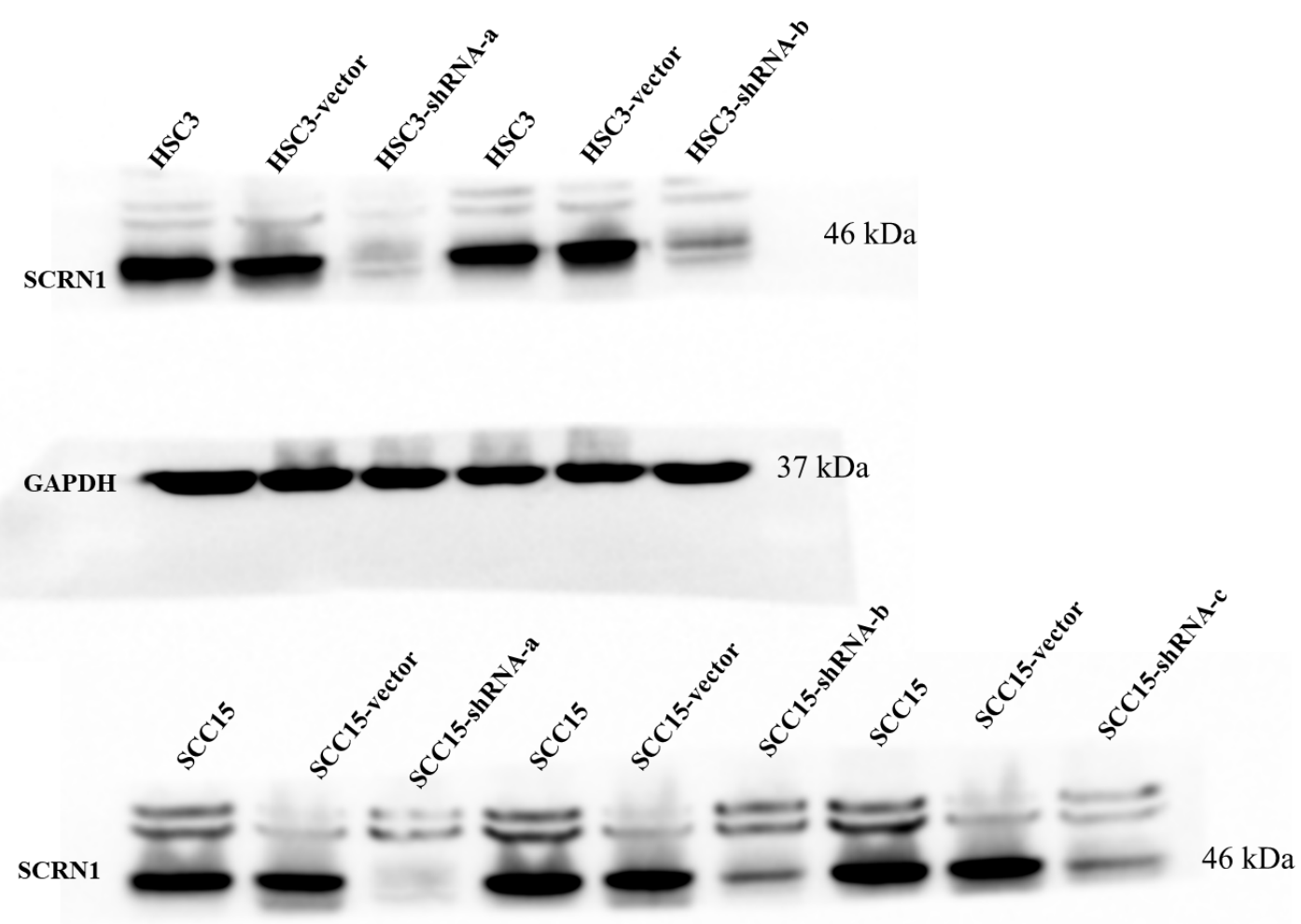

GAPDH

$37 \mathrm{kDa}$

\section{Figure 2}

Knockdown of SCRN1 suppresses proliferation, invasion and metastasis in OSCC cell lines (a) The expression levels of SCRN1 mRNA and protein in HSC3 and SCC15 cells transfected with three shorthairpin RNAs, namely shRNA-a, shRNA-b and shRNA-c, were evaluated via qPCR and WB. (b) SCRN1 knockdown suppressed HSC3 and SCC15 cell proliferation as determined by the CCK-8 assay. (c) Transwell migration assays assessed and quantified the migration of SCRN1 knockdown and control 
HSC3 and SCC15 cells. (d) Transwell migration assays detected and quantified the invasion of SCRN1knockdown HSC3 and SCC15 cells and control cells. Data presented as the mean \pm SD of three independent experiments. ${ }^{* *} p<0.01,{ }^{* \star *} p<0.001$ and ${ }^{* \star * *} p<0.0001$.

\section{Supplementary figure 3}

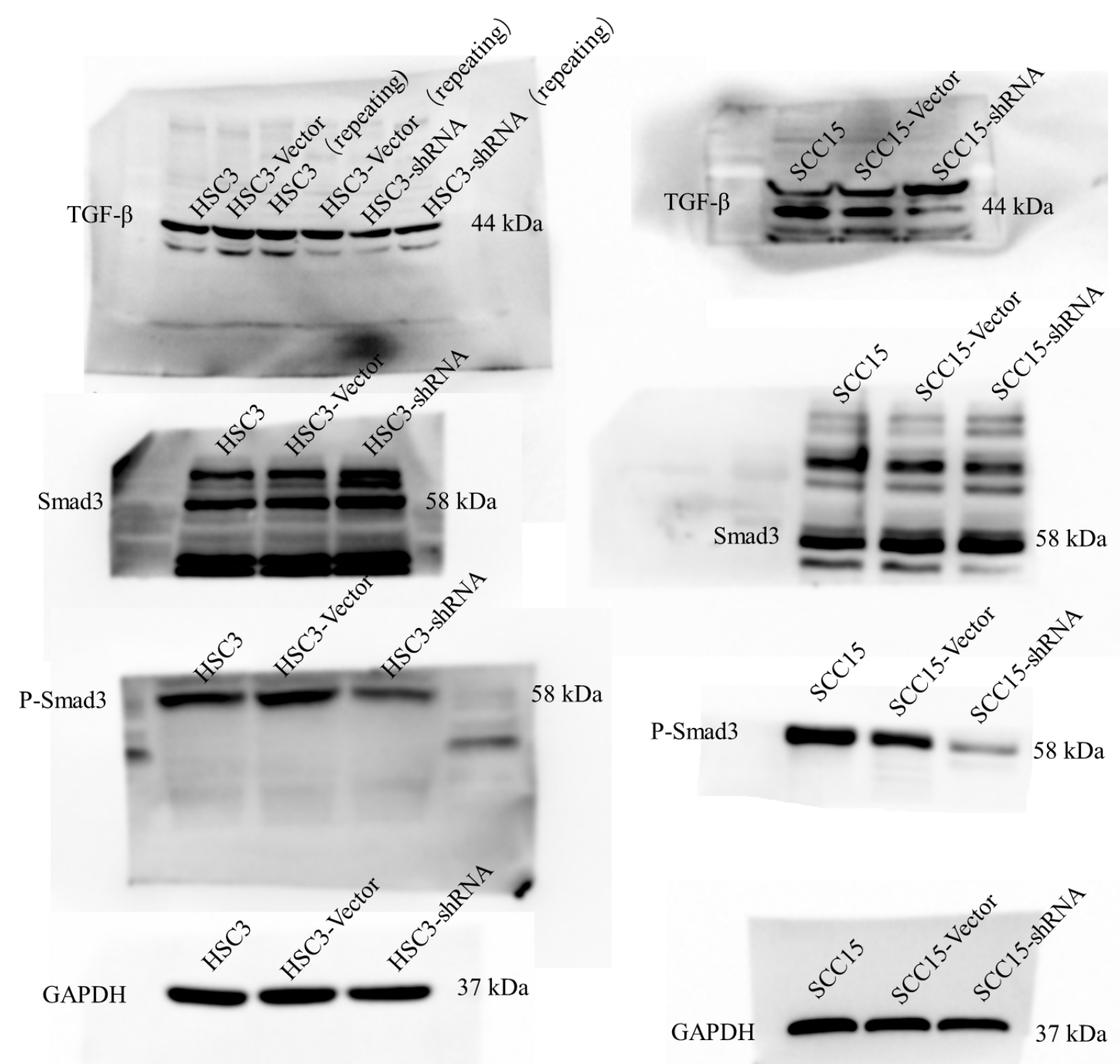

Figure 3

SCRN1 enhances MMP-9 exocytosis and activates the TGF- $\beta$ /Smad3 pathway (a) MMP-2 and MMP-9 protein secretion was detected by ELISA in SCRN1 knockdown SCC15 and HSC3 cells. Data presented as 
the mean $\pm S D$ of three independent experiments. ${ }^{* \star} p<0.01$, and ns $=$ no significant difference. (b) The levels of TGF- $\beta$ and $p$-Smad3 in HSC3 and SCC15 cells were determined by WB. GAPDH was used as a loading control. The ratios of p-Smad3 to the total levels were calculated based on the densities of the respective bands. Data presented as the mean \pm SD of three independent experiments. ${ }^{\star \star} p<0.01$. 